\title{
Video Article \\ Assessing Cerebral Autoregulation via Oscillatory Lower Body Negative Pressure and Projection Pursuit Regression
}

\author{
J. Andrew Taylor ${ }^{1,2}$, Can Ozan Tan ${ }^{1,2}$, J. W. Hamner ${ }^{2}$ \\ ${ }^{1}$ Department of Physical Medicine and Rehabilitation, Harvard Medical School \\ ${ }^{2}$ Cardiovascular Research Laboratory, Spaulding Hospital Cambridge
}

Correspondence to: J. Andrew Taylor at jandrew_taylor@hms.harvard.edu

URL: https://www.jove.com/video/51082

DOI: doi: $10.3791 / 51082$

Keywords: Medicine, Issue 94, cerebral blood flow, lower body negative pressure, autoregulation, sympathetic nervous system

Date Published: 12/10/2014

Citation: Taylor, J.A., Tan, C.O., Hamner, J.W. Assessing Cerebral Autoregulation via Oscillatory Lower Body Negative Pressure and Projection Pursuit Regression. J. Vis. Exp. (94), e51082, doi:10.3791/51082 (2014).

\section{Abstract}

The process by which cerebral perfusion is maintained constant over a wide range of systemic pressures is known as "cerebral autoregulation." Effective dampening of flow against pressure changes occurs over periods as short as $\sim 15 \mathrm{sec}$ and becomes progressively greater over longer time periods. Thus, slower changes in blood pressure are effectively blunted and faster changes or fluctuations pass through to cerebral blood flow relatively unaffected. The primary difficulty in characterizing the frequency dependence of cerebral autoregulation is the lack of prominent spontaneous fluctuations in arterial pressure around the frequencies of interest (less than $\sim 0.07 \mathrm{~Hz}$ or $\sim 15 \mathrm{sec}$ ). Oscillatory lower body negative pressure (OLBNP) can be employed to generate oscillations in central venous return that result in arterial pressure fluctuations at the frequency of OLBNP. Moreover, Projection Pursuit Regression (PPR) provides a nonparametric method to characterize nonlinear relations inherent in the system without a priori assumptions and reveals the characteristic non-linearity of cerebral autoregulation. OLBNP generates larger fluctuations in arterial pressure as the frequency of negative pressure oscillations become slower; however, fluctuations in cerebral blood flow become progressively lesser. Hence, the PPR shows an increasingly more prominent autoregulatory region at OLBNP frequencies of $0.05 \mathrm{~Hz}$ and below (20 sec cycles). The goal of this approach it to allow laboratory-based determination of the characteristic nonlinear relationship between pressure and cerebral flow and could provide unique insight to integrated cerebrovascular control as well as to physiological alterations underlying impaired cerebral autoregulation (e.g., after traumatic brain injury, stroke, etc.).

\section{Video Link}

The video component of this article can be found at https://www.jove.com/video/51082/

\section{Introduction}

The process by which cerebral perfusion is maintained constant over a wide range of systemic pressures is known as "cerebral autoregulation." Original observations of cerebral flow responses ${ }^{1}$ supported a counter-regulation against changes in arterial pressure that is of major importance for the daily regulation of cerebral perfusion. Although characterization of autoregulation was based on studies of sustained, controlled hypoand hypertension, ${ }^{2,3}$ it was recognized that pressure-induced changes in resistance are 'an oscillatory process' ${ }^{3}$ encompassing changes from 10 to 90 sec. $^{4}$ Moreover, within the past two decades, measurement of cerebral blood flow velocity on a beat-by-beat basis ${ }^{5}$ has shown that cerebral flow is regulated over periods as short as just a few heart beats. ${ }^{6,7}$ These beat-by-beat data suggest that effective dampening of flow against pressure changes occurs over periods as short as $\sim 15 \mathrm{sec}$ and it becomes progressively greater over longer time periods. ${ }^{8}$ Thus, the relationship between pressure and flow functions as a high pass filter ${ }^{7,9-12}$ wherein slower changes in blood pressure are effectively blunted and faster oscillations pass through relatively unaffected.

The primary difficulty in characterizing the frequency dependence of cerebral autoregulation is the lack of prominent spontaneous fluctuations in arterial pressure around the frequencies of interest (less than $\sim 0.07 \mathrm{~Hz}$ or $\sim 15 \mathrm{sec}$ ). Without sufficiently large pressure oscillations, one cannot accurately quantify the cerebral blood flow response. Our laboratory has dealt with this constraint by using a technique known as oscillatory lower body negative pressure (OLBNP). This creates caudal venous blood volume shifts proportional to the level of negative pressure in the tank due to reduced venous transmural pressure. When the negative pressure is applied at set intervals, the oscillations in central venous return result in arterial pressure fluctuations at the frequency of OLBNP. This approach has been used in several studies across different laboratories. $8,14-17$ This creates caudal venous blood volume shifts proportional to the level of negative pressure in the tank due to reduced venous transmural pressure. When the negative pressure is applied at set intervals, the oscillations in central venous return result in arterial pressure fluctuations at the frequency of OLBNP. This approach has been used in several studies across different laboratories. ${ }^{8,15-18}$

Even with an approach that can generate prominent fluctuations in arterial pressure around the frequencies of interest, there is a complicating factor: there is significant evidence of nonlinearity in cerebral autoregulation, especially at the lowest frequencies. ${ }^{8}$ Moreover, there is no strong theoretical guide as to the nature of nonlinearities present in cerebral autoregulation. Hence, we use an atheoretical, data driven method known as Projection Pursuit Regression (PPR) in our analysis. ${ }^{19} \mathrm{PPR}$ is a nonparametric method to characterize nonlinear relations inherent in a system without any a priori assumptions as to the nature of these nonlinearities. This is a decided advantage for capturing a system whose 
physiology is not yet defined by explicit nonlinear models. PPR reveals that the characteristic non-linearity of cerebral autoregulation resembles the "classic autoregulatory curve" first described by Lassen in 1959 (Figure 1). ${ }^{2,19}$ That is, cerebral blood flow remains relatively constant within a certain range of arterial pressure, but passively tracks in a linear fashion outside of this range. This shape becomes more apparent as arterial pressure fluctuation become slower. Hence, linear analysis is insufficient to fully interrogate cerebral autoregulation and reliance on linear techniques likely misses important information.

In this article we detail the approach to both data acquisition (laboratory use of OLBNP) and analysis (PPR) we use to characterize cerebral autoregulation in health and disease.

\section{Oscillatory Lower Body Negative Pressure (OLBNP)}

1. Equipment Setup

1. Electrocardiogram Lead II (ECG): Affix the three (or more) electrodes to the subject's torso for the monitoring of heart rate throughout the study.

2. Neoprene Skirt: Use a custom made neoprene skirt that seals the subject into lower body negative pressure chamber up to the iliac crest. Put it around the subject's chest before they are placed supine in the tank and ensure that the ECG signal is still adequate. Ensure that it is snug but not so tight as to restrict breathing.

3. Lower Body Negative Pressure Chamber: Have the subject lie supine on the bed and maneuver the LBNP chamber underneath them. If the LBNP chamber has an adjustable bicycle seat (to minimize movement artifact without counteracting the effect of the suction), make sure the subject is comfortably seated upon it. Use a custom made Plexiglas spacer cut to the subject's waist size to help seal the chamber. Seal the neoprene skirt around the LBNP chamber with duct tape.

4. LBNP Chamber Pressure: Connect the LBNP chamber to a standard pressure transducer. Calibrate the pressure transducer to mmHg.

5. Repeat Cycle Timer Attached to Mechanical Valve: Attach the custom built mechanical valve and repeat cycle timer to the LBNP chamber.

NOTE: A time delay relay attached to two motors that control a mechanical valve is used to alternate between negative pressure and ambient pressure. The time delay relay alternates voltage to the motors at a fixed interval to open and close a valve between the chamber and the vacuum. This creates an LBNP chamber pressure waveform that is roughly square wave in shape. Adjust the cycle time to the desired OLBNP frequency.

6. Variable Transformer and Vacuum: Attach a standard household vacuum cleaner to the mechanical valve. Plug the vacuum into a variable transformer that allows the voltage to the vacuum to be controlled. Turn on the vacuum cleaner and adjust the variable transformer until the target LBNP pressure (e.g., $30 \mathrm{mmHg}$ ) is achieved.

7. Arterial Blood Pressure: Attach non-invasive photoplethysmographic arterial pressure cuffs (e.g., Portapres, Finapres) to the finger(s) of one hand. Ensure accuracy by comparing pressure to oscillometric pressures from the brachial artery of the opposite arm.

8. $2 \mathrm{MHz}$ Transcranial Doppler and Probe Fixation Device

1. Use a $2 \mathrm{MHz}$ pulse wave Doppler probe to insonate the $\mathrm{M} 1$ segment of the middle cerebral artery at the temple (i.e., the transtemporal window).

2. Alter probe angle, insonation depth $(\sim 55 \mathrm{~mm})$, gain, and transmission power to maximize the spectral intensity of the signal.

3. Fix the Doppler probe in place using a fixation device that has no back (i.e., not a headband) so that movement artifact is not introduced into the signal as the volunteer moves with negative pressure oscillations.

NOTE: Cerebral blood flow can be measured unilaterally or bilaterally, but no difference in cerebral autoregulation is expected between hemispheres unless a localized injury like stroke or traumatic brain injury is present. ${ }^{20}$

9. Expired $\mathrm{CO}_{2}$ : Use a nasal cannula attached to an infrared $\mathrm{CO}_{2}$ analyzer to monitor expired $\mathrm{CO}_{2}$ and instruct the subject to breathe only through their nose. Given the profound effect arterial $\mathrm{CO}_{2}$ has on cerebral blood flow, ${ }^{21}$ monitor $\mathrm{CO}_{2}$ throughout every study.

2. Data Acquisition

1. Set up the analog to digital conversion of arterial pressure, cerebral blood flow, LBNP chamber pressure, and expired $\mathrm{CO}_{2}$ to acquire at a minimum of $50 \mathrm{~Hz}$ per channel. Acquire ECG at $1 \mathrm{kHz}$.

NOTE: While subsequent analysis deals with much lower frequency information $(\leq 0.07 \mathrm{~Hz})$, it is critical to monitor the quality of the signals being acquired during a study. A sampling rate of $50 \mathrm{~Hz}$ will allow accurate visualization of blood pressure and cerebral blood flow for the detection of artifact.

3. Oscillatory LBNP Protocol

1. Turn on vacuum and ensure tank pressure is stable at $-30 \mathrm{mmHg}$

2. Set repeat cycle timer to $33 \mathrm{sec}$ for $0.03 \mathrm{~Hz}$ OLBNP.

3. Adjust Doppler probe(s) to ensure optimal signal.

4. Acquire data for at least 15 cycles $(500 \mathrm{sec}$ at $0.03 \mathrm{~Hz})$ to ensure sufficient confidence in PPR estimates. If time permits, collect more data than this as it will further improve the signal-to-noise ratio.

5. Repeat the above steps for any frequencies between $0.03 \mathrm{~Hz}-0.08 \mathrm{~Hz}$ by changing the repeat cycle timer duration. NOTE: Apply frequencies in order but randomly vary the starting frequency between subjects.

\section{Projection Pursuit Regression (PPR)}

1. Data Preprocessing

1. Decimation and Low-pass Filtering 
1. Open Matlab. Type the command "data = resample(data, $1, \mathrm{SR} / 5)$ " (where SR is the original sampling rate) to decimate the arterial pressure and cerebral blood flow to $5 \mathrm{~Hz}$.

NOTE: Optionally, low-pass filter (19 $9^{\text {th }}$ order Chebyshev Type II) with a cutoff of $0.4 \mathrm{~Hz}$. The filtering is redundant, given subsequent processing, but creates mean waveforms that don't rely on peak detection of the sometimes noisy arterial pressure and cerebral blood flow signals.

2. Artifact Removal

1. Using the original non-decimated waveforms as a guide, remove any sections of the signals with artifacts and linearly interpolate. If these sections account for more than $10 \%$ of the recording period, discard the recording entirely. NOTE: At this point, the waveforms are suitably processed for traditional linear approaches such as transfer function analysis.

3. Band-pass Filtering

1. In Matlab, type: $[B, A]=$ cheby $1(1,1,[F-0.005 F+0.005] /(S R d / 2))$ data $=$ filtfilt( $B, A$, detrend(data, 'linear') to band-pass filter the pressure and flow in a $\pm 0.005 \mathrm{~Hz}$ band ( $1^{\text {st }}$ order Chebyshev Type I with $1 \mathrm{~dB}$ of pass band ripple) around the frequency of OLBNP (Figure 2) where $\mathrm{F}$ is the dominant OLBNP frequency, SRd is the decimated sampling rate (5 Hz after step 2.1.1), and "data" is the decimated signal (arterial pressure or flow).

NOTE: This minimizes potential interference and increases the signal-to-noise ratio in subsequent PPR analysis. Although the dominant arterial pressure fluctuation occurs at the oscillatory frequency of lower body negative pressure, random noise in the signals may interfere with the derivation of pressure-flow relationships. Results without band-pass filtering will be qualitatively similar but the percent variance explained (i.e., $R^{2}$ ) will be lower. ${ }^{19}$

2. Projection Pursuit Regression Estimation

NOTE: Using the built-in function 'ppr' in R Language and Environment for Statistical Computing, and/or via custom-written functions in other platforms, generate a single ridge function $(M=1)$ for the arterial pressure-cerebral flow relation.

1. In Matlab, enter the command "CVLabPPR(pressure,flow)". Enter the Study ID as XXXYYY, where XXX is the 3-letter study code and YYY is the three numeric characters for subject ID. Enter the Study Date in the following format: YYYY-MM-DD. Enter the numeric measurement \# (e.g., "1" for day 1).

2. Enter the APM (enter FP for finapress or AL for art-line). Enter the Vessel (MCA, ACA, or PCA). Enter "y" or " $n$ " to the query "Do you have right MCA measurements?" Enter "y" or " $n$ " to the query "Do you have left MCA measurements?"

NOTE:

$y_{t}=f\left(x_{t}\right):=\sum_{m=1}^{M} \alpha_{m} k_{m}\left(\sum_{i} \gamma_{i}^{m} x_{t-i}\right)$

For each input ( $x_{t}$ - arterial blood pressure) and output ( $y_{t}-$ cerebral blood flow) a linear autoregressive transfer function (Eq 1. the term within the parentheses) is passed through nonparametric kernel functions ( $k_{m}$; called 'ridge functions') that are determined by minimizing the mean squared error. Projection pursuit regression can include more than one ridge function (i.e., $M>1)$. However, though it will reduce the mean squared error, it may obscure the interpretation of ridge functions due to potential interactions between them. Because the primary purpose is to obtain a relation between arterial pressure and cerebral blood flow that can be interpreted physiologically, PPR should be limited to only one ridge function $(M=1)$.

3. Piecewise Linear Parameterization. Parameterize the ridge function as a piecewise linear function for subsequent statistical analysis (Figure 3). For Matlab, use Bruno Luong's Free-knot spline approximation. Enter the command "BSFK(x, y, k, nknots)" where k=2 for a linear fit and nknots $=3$ for three regions.

NOTE: This identifies those points where the arterial pressure-cerebral flow relationship changes, and the ranges wherein the relationship is approximately linear. Figure 3 shows a schematic of the results. The gain (i.e., the linear slope) of the pressure-flow relation within each region provides a measure of the effectiveness of cerebral autoregulation within that region. A lower gain indicates more effective counter-regulation of pressure fluctuations whereas higher gains indicate more passive flow responses to pressure changes.

\section{Representative Results}

OLBNP amplitudes from $10 \mathrm{mmHg}^{22}$ up to $120 \mathrm{mmHg}^{17}$ have been used to augment arterial pressure fluctuations, but $30 \mathrm{mmHg}$ OLBNP is sufficient ${ }^{23,24}$ and not beyond the regulatory capacity of the cerebrovasculature. ${ }^{17}$ This level of OLBNP results in blood pressure oscillations that are about $15-20 \mathrm{mmHg}$ in magnitude at $0.03 \mathrm{~Hz}$, which is not greater than the blood pressure changes occurring when going from seated to standing. ${ }^{25}$ There are some limitations to the range within which OLBNP can generate arterial pressure fluctuations. Autoregulation is only active at $\sim 0.07 \mathrm{~Hz}$ and slower, so the upper limit is not an issue. However the difficulty in generating low frequency oscillations below $0.03 \mathrm{~Hz}$ is that the cardiovascular system counter-regulates against the LBNP-induced arterial pressure changes before the cycle is finished. As Figure 4 shows, at $0.025 \mathrm{~Hz}$ OLBNP we actually see the largest peak in the arterial pressure oscillations at $0.05 \mathrm{~Hz}$. While the frequency response of cerebral autoregulation can be characterized from $0.03 \mathrm{~Hz}-0.08 \mathrm{~Hz}$ to define the time scales within which autoregulation is active, ${ }^{23,24} 0.03 \mathrm{~Hz}$ and 0.08 $\mathrm{Hz}$ OLBNP are sufficient since they represent a range of autoregulatory function (i.e., a pronounced autoregulatory region to none or a modest one).

OLBNP generates larger fluctuations in arterial pressure as the frequency of negative pressure oscillations become slower. Figure 5 shows the arterial pressure and consequent cerebral blood flow fluctuations with OLBNP from $0.08 \mathrm{~Hz}$ ( $12.5 \mathrm{sec}$ cycles) to $0.03 \mathrm{~Hz}$ ( $33 \mathrm{sec}$ cycles) At the higher frequencies, cerebral blood flow fluctuates in concert with arterial pressure. The PPR demonstrates this; there is a proportional linear relation between arterial pressure and cerebral blood flow at the higher frequencies of $0.08 \mathrm{~Hz}, 0.07 \mathrm{~Hz}(14 \mathrm{sec}$ cycles), and $0.06 \mathrm{~Hz}$ (16.6 sec cycles). At slower frequencies of OLBNP, though arterial pressure fluctuations become larger, fluctuations in cerebral blood flow are progressively more effectively dampened. Hence, the PPR shows an increasingly more prominent autoregulatory region at OLBNP frequencies 
from $0.05 \mathrm{~Hz}$ (20 sec cycles), to $0.04 \mathrm{~Hz}$ (25 sec cycles), to $0.03 \mathrm{~Hz}$. In the example shown, at $0.03 \mathrm{~Hz}$, the PPR curve clearly resembles the "classic autoregulatory curve" described by Lassen (Figure 1). We have previously shown that this observation cannot be explained simply by the increase in magnitude of arterial pressure fluctuations as the frequency of oscillations become slower. We have previously applied PPR to data from 48 individuals during different magnitudes of OLBNP (thus, different magnitude of pressure fluctuations). ${ }^{19}$ While we did not explicitly explore a potential relation between autoregulatory range and the magnitude of pressure fluctuations, we reported that the variation in autoregulatory range was only $\sim 6 \%$. Thus, our prior results clearly show that the change in PPR curve with frequency cannot be fully explained by a change in the magnitude of pressure fluctuations. In the same study, we assessed whether the PPR characterization of autoregulation is reproducible across separate sessions. This analysis showed that the slope of the autoregulatory range during $0.03 \mathrm{~Hz} O \mathrm{LBNP}$ did not change (Lin's Concordance $=0.96, p<0.001$ ) and thus the nonlinear pressure-flow relation is consistent across study days.

Although the cerebrovascular bed is well innervated by sympathetic nerve fibers, their role in autoregulation has not been widely accepted. ${ }^{26}$ Therefore, some of our previous work explored the potential role of the sympathetic nervous system in cerebrovascular autoregulation. ${ }^{24}$ We found a clear role for the sympathetic system in regulating cerebral flow, but we were not able to characterize how the relationship changed with removal of sympathetic effects due to the limitations of linear methods for characterizing autoregulation. Figure 6 shows the results from PPR application to data before (baseline) and after sympathetic blockade during $0.05 \mathrm{~Hz}$. The overall curve becomes markedly more linear. Moreover, PPR analysis of $0.03 \mathrm{~Hz}$ data where autoregulation is most apparent showed that the range of the autoregulatory region remains unchanged, but the slope within that region increases, reflecting less effective autoregulation (Figure 7).

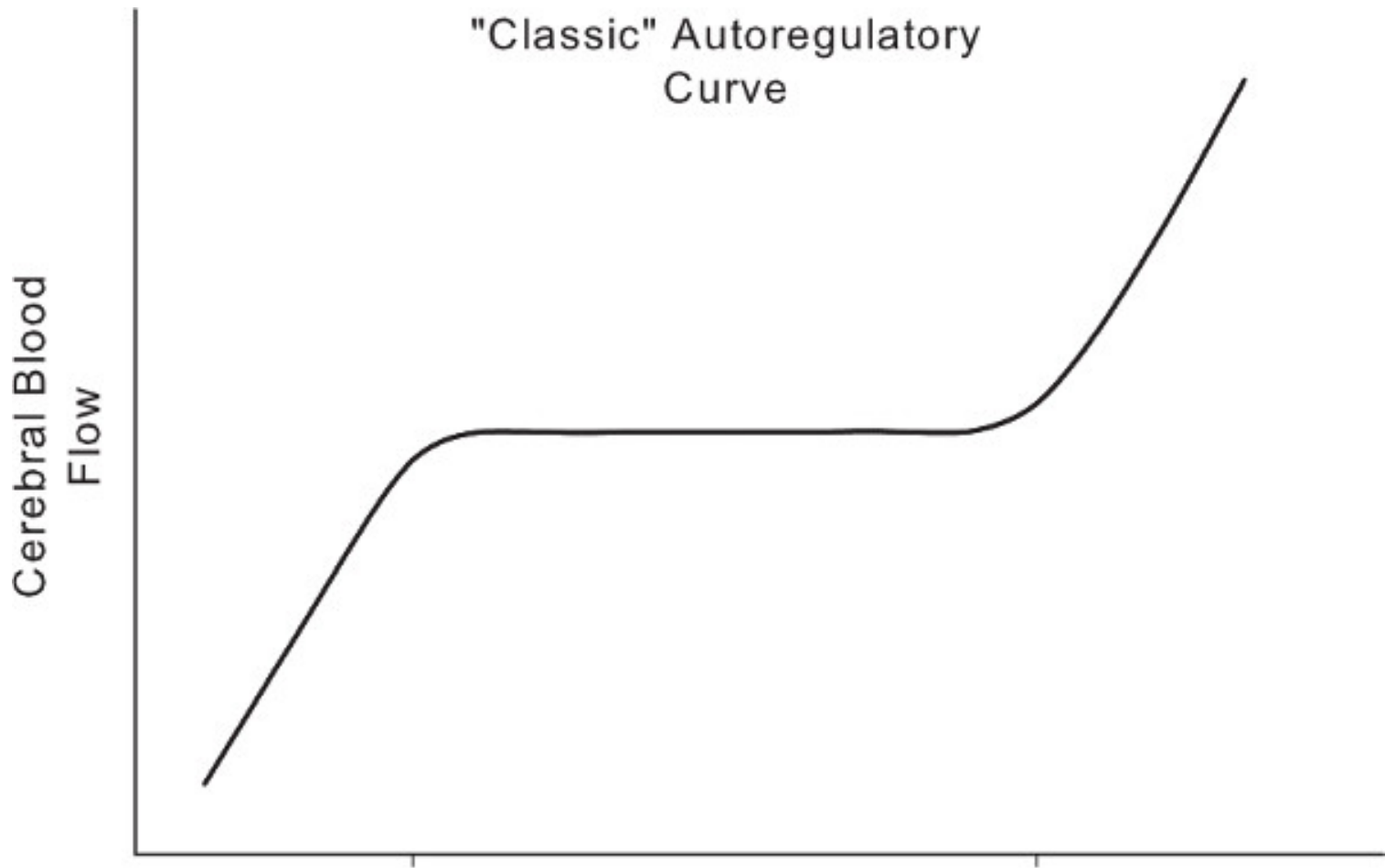

60

\section{Arterial Pressure $(\mathrm{mmHg}$ )}

Figure 1. The 'classic' autoregulatory curve derived from the relationship between static increases and decreases in pressure and steady state cerebral blood flow. A region of unchanging flow despite changing pressure $(i . e .$, slope $=0)$ is bounded by regions wherein increasing and decreasing pressures result in proportional cerebral blood flow changes. 

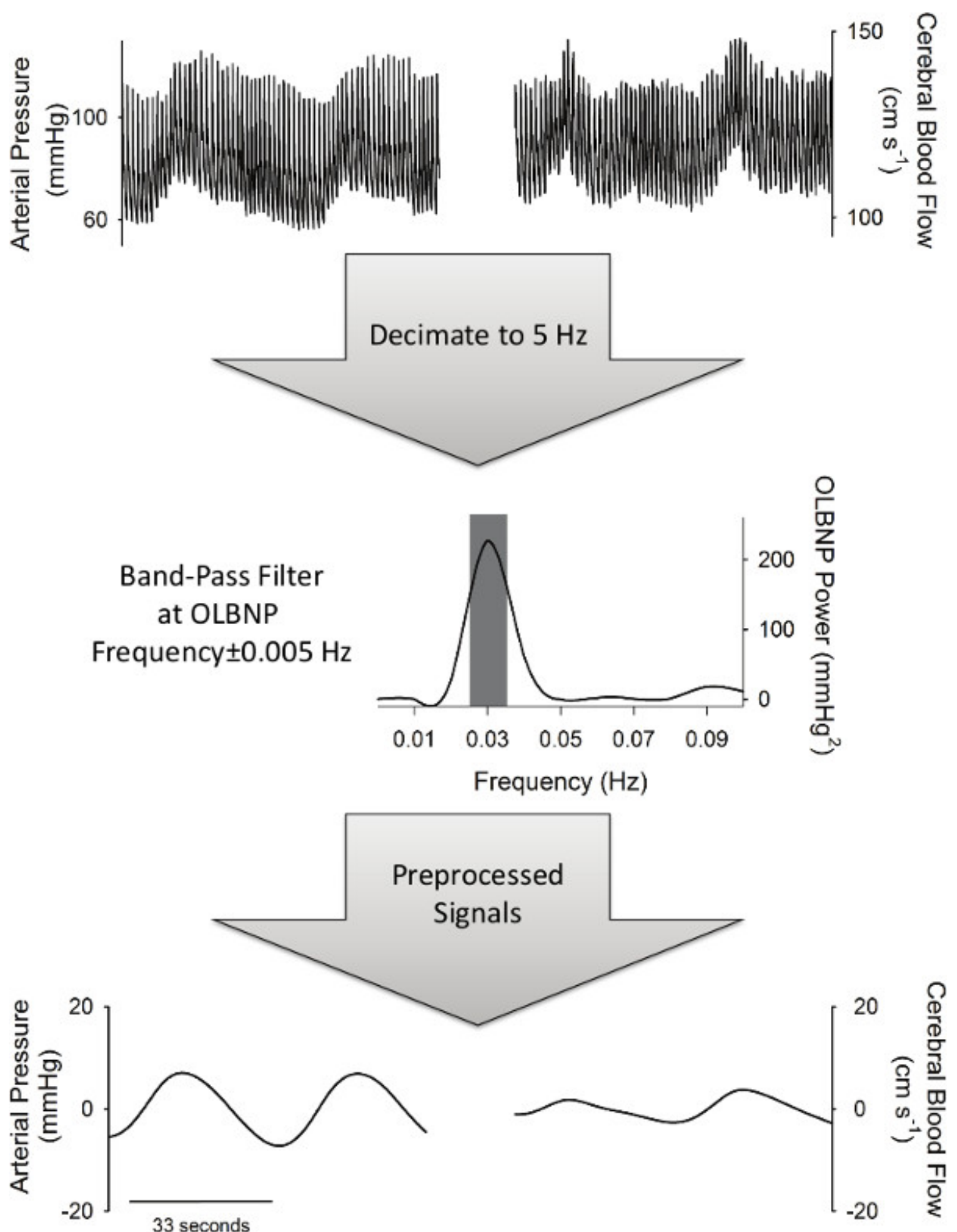

Figure 2. The preprocessing necessary to perform PPR analysis. Signals are first decimated to $5 \mathrm{~Hz}$ and then band-pass filtered at the frequency of OLBNP $( \pm 0.005 \mathrm{~Hz})$. 


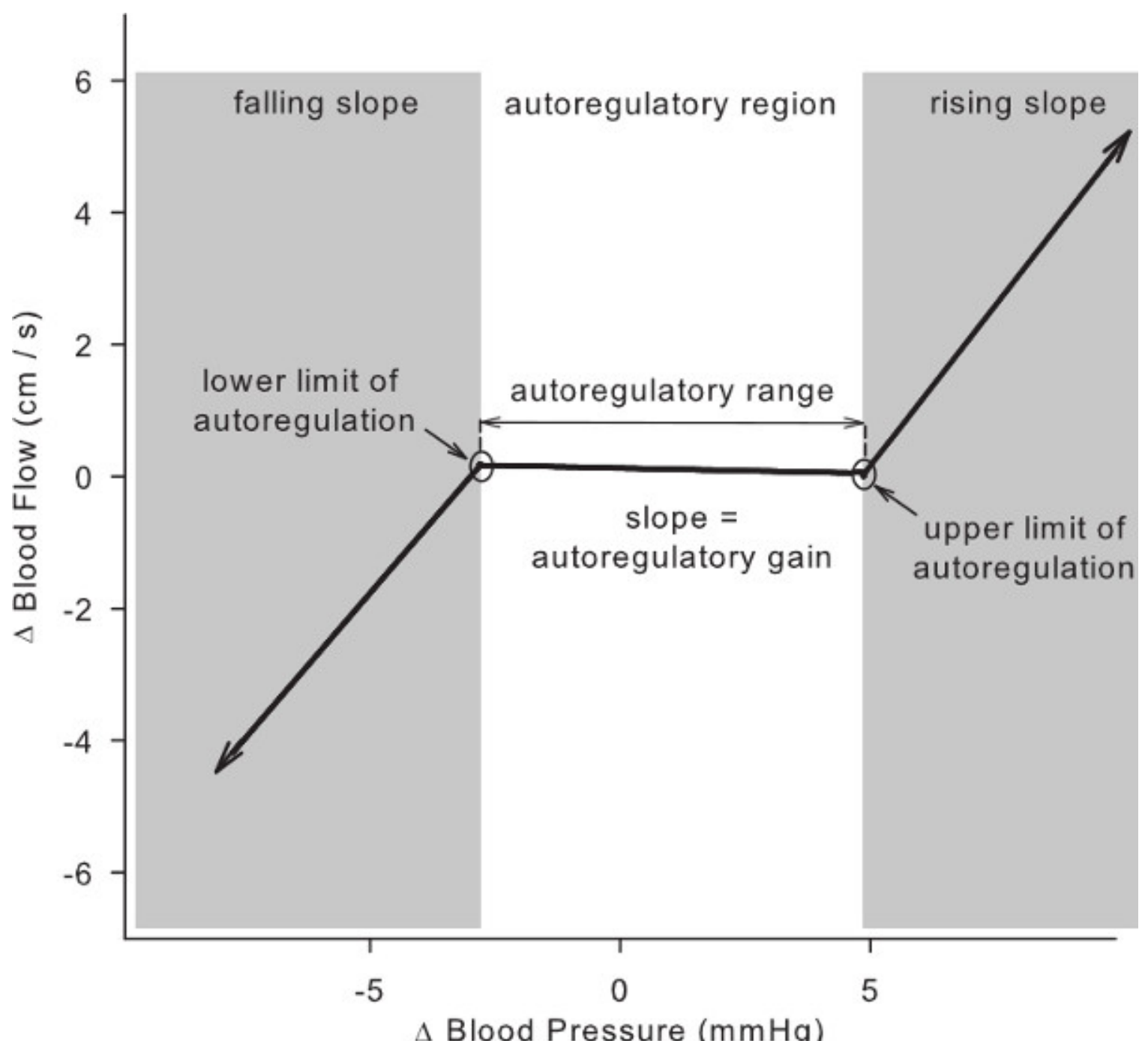

Figure 3. Parameters of the cerebral autoregulation curve derived from PPR analysis of arterial pressure and cerebral blood flow during $0.03 \mathrm{~Hz}$ OLBNP. 


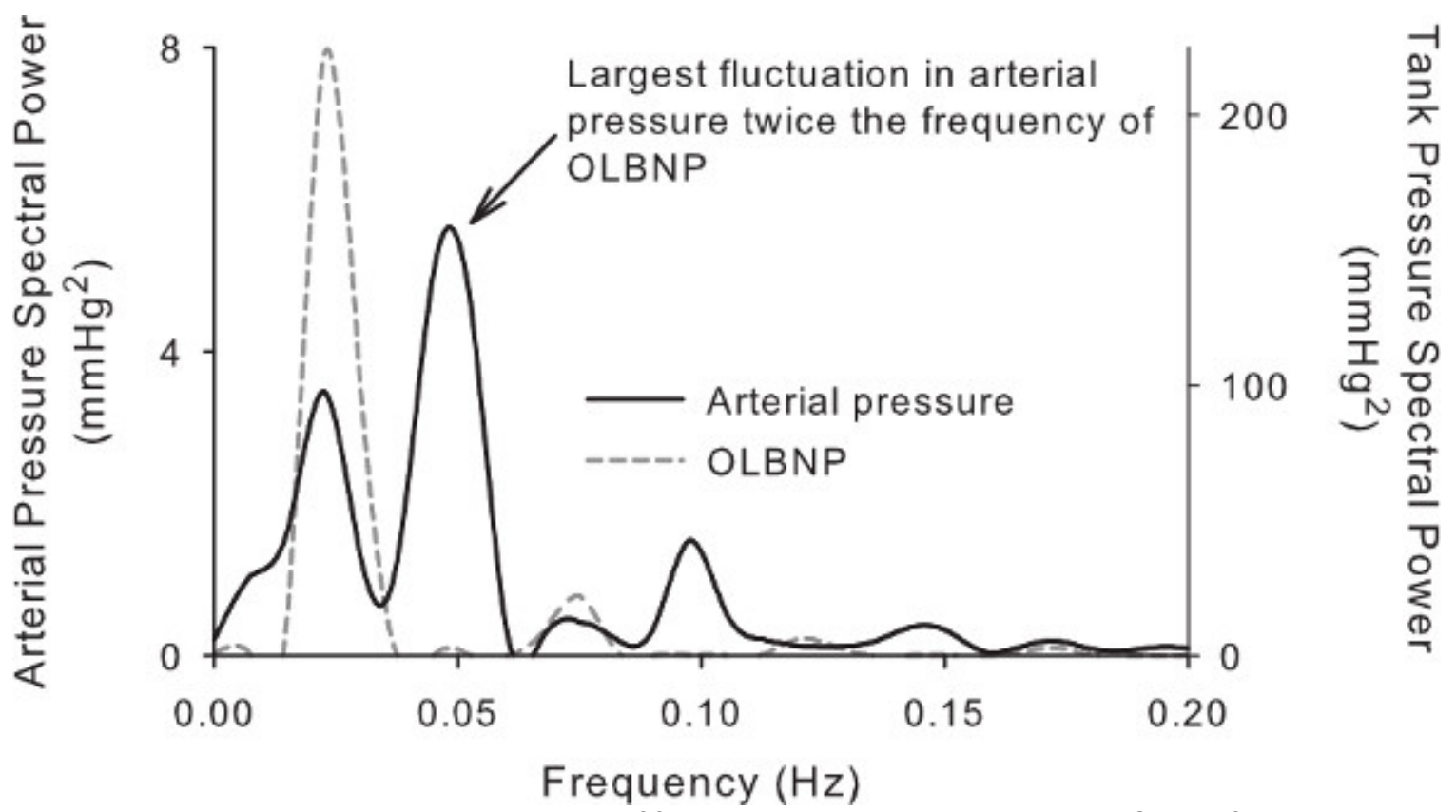

Figure 4. Power spectrum shows the magnitude of fluctuations in arterial pressure when OLBNP frequency is below $0.03 \mathrm{~Hz}(33-$ second cycle). Note that there are two large peaks in the arterial pressure spectral power at 0.025 and $0.05 \mathrm{~Hz}(40$ and $20 \mathrm{sec}$ cycles), however there is only a single peak in the LBNP spectral power at $0.025 \mathrm{~Hz}$. Moreover, the largest fluctuation in pressure is at $0.05 \mathrm{~Hz}$ and would confound the interpretation of the cerebral blood flow responses. 


\section{$\begin{array}{llllll}0.03 \mathrm{~Hz} & 0.04 \mathrm{~Hz} & 0.05 \mathrm{~Hz} & 0.06 \mathrm{~Hz} & 0.07 \mathrm{~Hz} & 0.08 \mathrm{~Hz}\end{array}$}

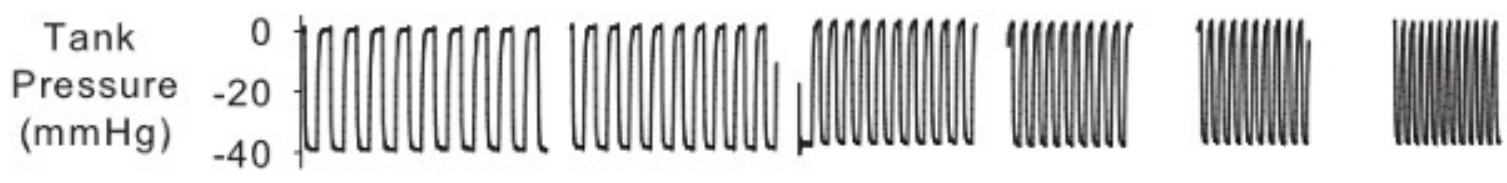

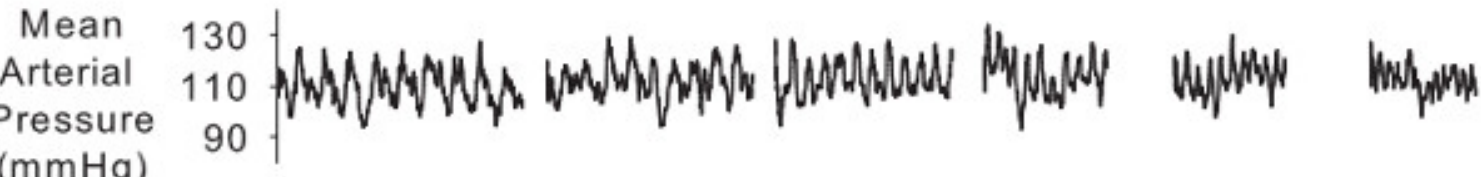 \\ $(\mathrm{mmHg})$}
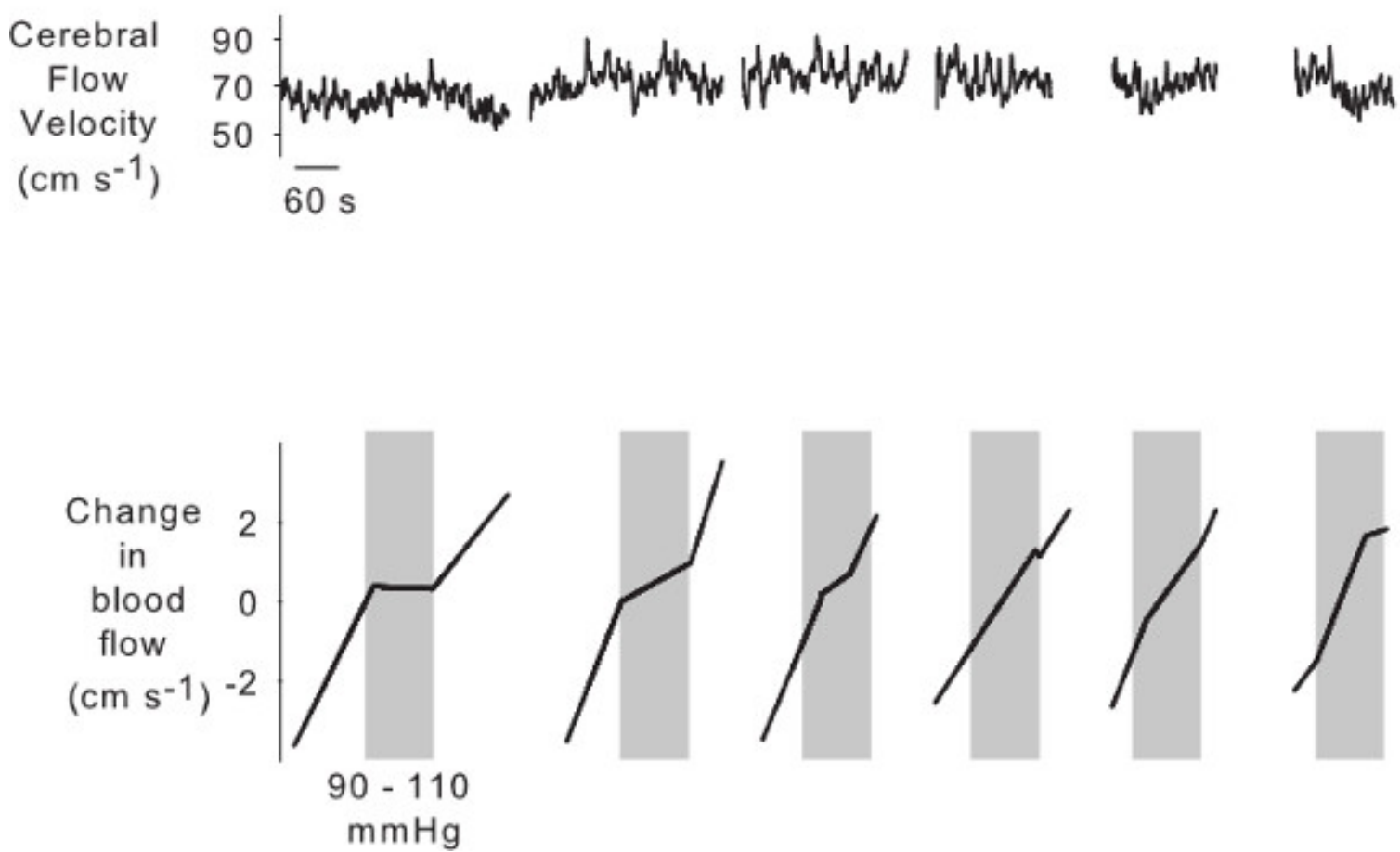

Figure 5. Example of the effects of OLBNP from 0.08 to $0.03 \mathrm{~Hz}$ on arterial pressure and cerebral blood flow. Arterial pressure fluctuations become larger with slower OLBNP whereas cerebral blood flow fluctuations become smaller. This autoregulatory function is described by the results of the PPR analysis shown in the bottom panels. The autoregulatory region in cerebral blood flow becomes progressively more pronounced with slower OLBNP. 


\section{Baseline}

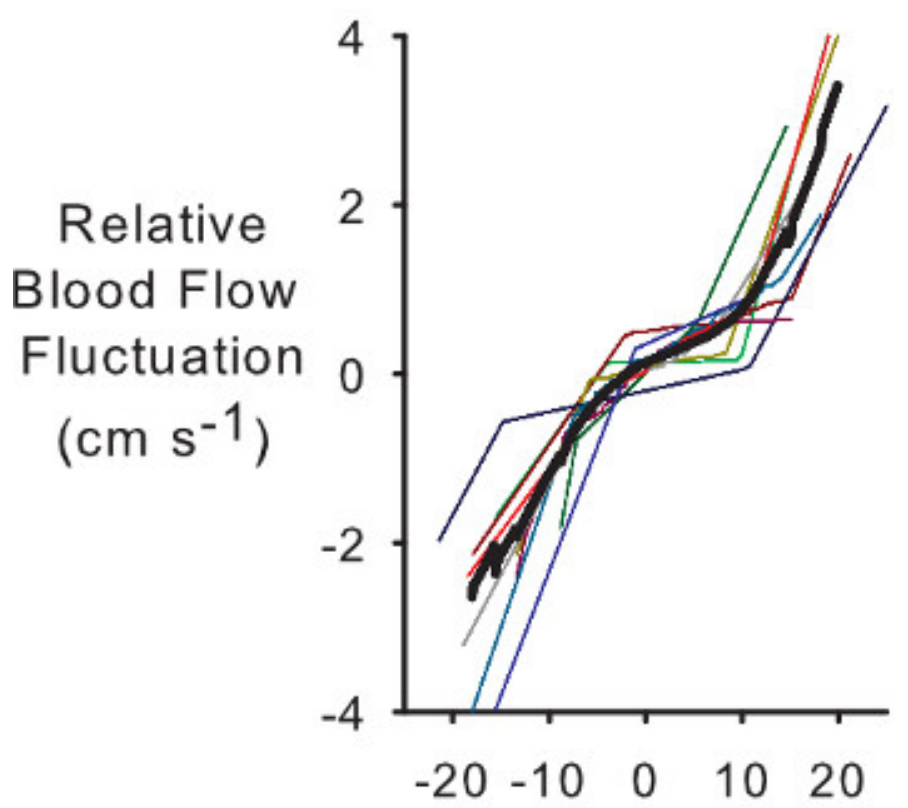

\section{Sympathetic blockade}

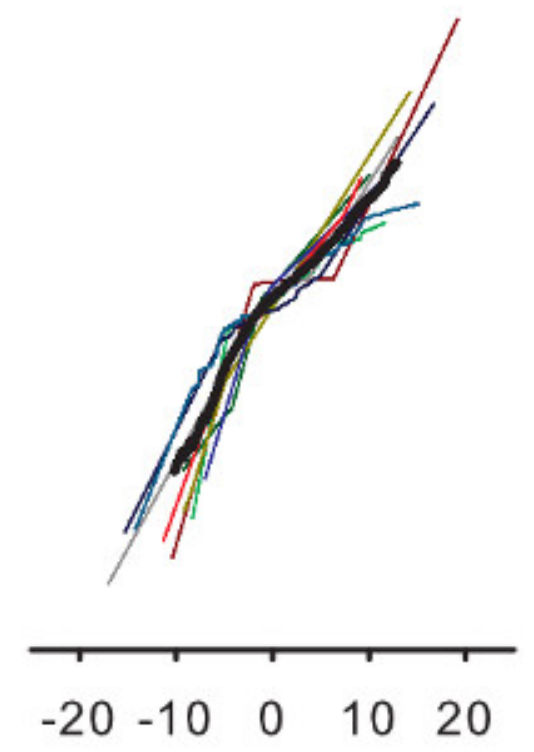

Relative Blood Pressure Fluctuation $(\mathrm{mmHg}$ )

Figure 6. Individual and averaged PPR autoregulatory curves from $0.05 \mathrm{~Hz}$ OLBNP data in subjects before (baseline) and after sympathetic blockade. Note that the loss of the narrow autoregulatory region after sympathetic blockade.

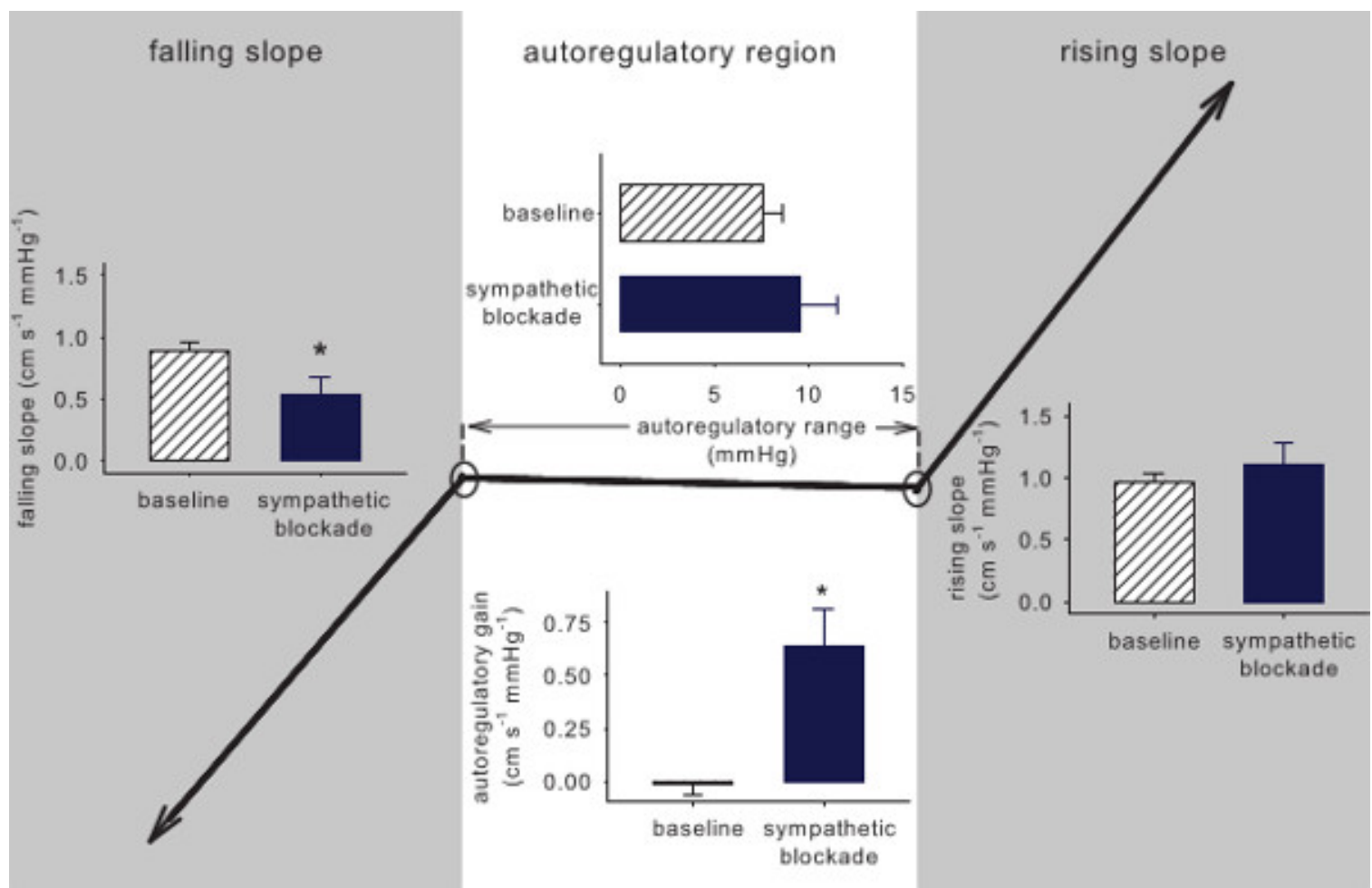

Figure 7. Average of the PPR parameters from $0.03 \mathrm{~Hz}$ OLBNP data before and after sympathetic blockade. Sympathetic blockade had a pronounced effect on the cerebral autoregulation curve within the autoregulatory range, markedly increasing the slope (i.e., more proportional cerebral flow changes with pressure changes). 


\section{Discussion}

Precisely defining input-output relations can require that the input (in this case, pressure) actively changes across a sufficiently wide range to observe the output response. However, spontaneously occurring pressure fluctuations are extremely inconsistent and small in amplitude within the frequencies of cerebral autoregulation. ${ }^{27}$ This is the reason that spontaneous changes in pressure and flow show a relationship with periods of high correlation and periods of extremely low correlation and that oscillations in cerebral blood flow seemingly appear with no apparent arterial pressure drive. ${ }^{28}$ OLBNP $^{22}$ provides a critical technique to create consistent arterial pressure oscillations of varying frequency and amplitude to assess cerebral blood flow responses. Although there may be other approaches that might provide a similar probe, this approach allows for rigorous testing of the frequency- and/or amplitude-dependent relationship between arterial pressure and cerebral blood flow velocity.

Prior research exploring potential measurement tools for cerebral autoregulation have used linear models of the relation between arterial pressure and cerebral blood flow (e.g., transfer function analysis). A close linear relation between pressure and flow changes with no dampening is observed when pressure oscillations are relatively fast, i.e., $>\sim 10 \mathrm{sec}$. However, slower oscillations ( $>\sim 20$ sec) engender a relation between pressure and flow that becomes progressively less linearly related. ${ }^{8,24}$ If the relation is not highly linearly related (low $\mathrm{R}^{2}$, low crossspectral coherence) one cannot have any confidence in the accuracy of linear measures such as transfer function gain and phase. The lack of linear relation indicates the presence of important nonlinearities that are characteristic of cerebral autoregulation. In fact, by its very nature, autoregulation is not amenable to characterization via linear approaches; linear approaches can indicate presence or absence of autoregulation, but cannot describe its characteristics and its effectiveness.

There are methods that are comparable to linear methods in their simplicity but that can assess nonlinear relationships between input (pressure) and output (flow) variables. Projection pursuit regression is simply a nonparametric, atheoretical, multiple regression method ${ }^{29,30}$ that does not posit an a priori model or assume linearity in the input-output relation. These are clear advantages for characterizing a system that is incompletely understood. However, it should be noted that using more than one ridge function will increase the percent variance explained but at the expense of obscuring physiologic interpretation of the characteristic relationships. Therefore, it is recommended that projection pursuit regression be limited to only one ridge function. Nonetheless, the PPR approach outlined with a single ridge function can explain a significant portion of the variance in the relation between arterial pressure and cerebral blood flow and reveal the characteristic nonlinear relation that is consistent across individuals.

\section{Limitations and Possible Modifications}

Oscillatory lower body negative pressure requires specific and obtrusive equipment and procedures and so is not appropriate for clinic based assessments. It is possible that resting recordings of sufficient length could provide adequate data for PPR analysis of cerebral autoregulation. However, previous work showed that projection pursuit regression of resting data performs significantly worse than analysis of $0.03 \mathrm{~Hz}$ OLBNP data. Although pressure-flow relationships quantified at rest and during $0.03 \mathrm{~Hz}$ OLBNP are related, ${ }^{19}$ the modest correspondence simply suggests that the pressure-flow relationships estimated at rest may not reliably reflect those derived from $0.03 \mathrm{~Hz}$ OLBNP. One solution may be to generate consistent and larger amplitude pressure fluctuations within the frequencies of autoregulation via slow, deep eucapnic breathing or repeated squat-stand maneuvers. These methods have been shown to generate reliably large pressure fluctuations that may provide changes across a sufficiently wide range to observe cerebral blood flow responses. ${ }^{31,32}$

Though on average, projection pursuit regression can explain a significant amount of the relationship between arterial pressure and cerebral flow fluctuations, explained variance may be low in a few cases $\left(\sim 6 \%{ }^{19}\right)$. Low performance could derive, for example, from breathing patterns if frequency and tidal volume are not controlled. However, every physiological test has some aberrant observations, and this approach is not an exception. Poor measurements in $\sim 1$ of 20 observations should not undermine the potential utility of the approach.

\section{Future Applications / Conclusions}

The characteristic pressure-flow relationship may be altered in some pathophysiologic conditions, such as stroke ${ }^{33}$ and traumatic brain injury. ${ }^{34}$ If accurate relations could be acquired in the clinical setting, projection pursuit regression of cerebral autoregulation may have broader application and be useful as an assessment tool where OLBNP is not available. It is possible that simple maneuvers (e.g., deep breathing, thigh cuff, sit-to-stand) and/or longer duration resting recordings could result in pressure-flow relationship that can be sued to derive cerebral autoregulation comparable to OLBNP data. Nonetheless, laboratory-based determination of different regulatory systems and their contribution to the nonlinearities of autoregulation could provide unique insight to cerebrovascular control, and allow diagnosis of pathophysiological alterations in cerebral autoregulation (e.g., after traumatic brain injury).

\section{Disclosures}

The authors declare that they have no competing financial interests.

\section{Acknowledgements}

This research was supported by National Heart, Lung, and Blood Institute Grant HL-093113.

\section{References}

1. Strandgaard, S., \& Paulson, O. B. Cerebral autoregulation. Stroke. 15, 413-416 (1984)

2. Lassen, N. A. Cerebral blood flow and oxygen consumption in man. Physiol. Rev. 39, 183-238 (1959). 
3. Symon, L., Held, K., \& Dorsch, N. W. A study of regional autoregulation in the cerebral circulation to increased perfusion pressure in normocapnia and hypercapnia. Stroke. 4, 139-147 (1973).

4. Rapela, C. E., \& Green, H. D. Autoregulation of Canine Cerebral Blood Flow. Circ. Res. 15, SUPPL:205-212 (1964).

5. Aaslid, R., Markwalder, T. M., \& Nornes, H. Noninvasive transcranial Doppler ultrasound recording of flow velocity in basal cerebral arteries. J. Neurosurg. 57, 769-774, doi:10.3171/jns.1982.57.6.0769 (1982).

6. Aaslid, R., Lindegaard, K. F., Sorteberg, W., \& Nornes, H. Cerebral autoregulation dynamics in humans. Stroke. 20, 45-52 (1989).

7. Newell, D. W., Grady, M. S., Sirotta, P., \& Winn, H. R. Evaluation of brain death using transcranial Doppler. Neurosurgery. 24, 509-513 (1989).

8. Hamner, J. W., Cohen, M. A., Mukai, S., Lipsitz, L. A., \& Taylor, J. A. Spectral indices of human cerebral blood flow control: responses to augmented blood pressure oscillations. J. Physiol. 559, 965-973, doi:10.1113/jphysiol.2004.066969 jphysiol.2004.066969 [pii] (2004).

9. Blaber, A. P. et al. Complexity of middle cerebral artery blood flow velocity: effects of tilt and autonomic failure. Am J Physiol. 273, H2209-2216 (1997).

10. Diehl, R. R., Linden, D., Lucke, D., \& Berlit, P. Spontaneous blood pressure oscillations and cerebral autoregulation. Clin. Auton. Res. 8, 7-12 (1998).

11. Panerai, R. B., Rennie, J. M., Kelsall, A. W., \& Evans, D. H. Frequency-domain analysis of cerebral autoregulation from spontaneous fluctuations in arterial blood pressure. Med. Biol. Eng. Comput. 36, 315-322 (1998).

12. Zhang, R., Zuckerman, J. H., \& Levine, B. D. Deterioration of cerebral autoregulation during orthostatic stress: insights from the frequency domain. J. Appl. Physiol. 85, 1113-1122 (1998).

13. Wolthuis, R. A., Bergman, S. A., \& Nicogossian, A. E. Physiological effects of locally applied reduced pressure in man. Physiol. Rev. 54, 566-595 (1974).

14. Esch, B. T., Scott, J. M., \& Warburton, D. E. Construction of a lower body negative pressure chamber. Adv. Physiol. Educ. 31, 76-81, doi:31/1/76 [pii] 10.1152/advan.00009.2006 (2007).

15. Brown, C. M., Dutsch, M., Ohring, S., Neundorfer, B., \& Hilz, M. J. Cerebral autoregulation is compromised during simulated fluctuations in gravitational stress. Eur. J. Appl. Physiol. 91, 279-286, doi:10.1007/s00421-003-0965-5 (2004).

16. Hidaka, I. et al. Noise-enhanced heart rate and sympathetic nerve responses to oscillatory lower body negative pressure in humans. $J$. Neurophysiol. 86, 559-564 (2001).

17. Tzeng, Y. C., Chan, G. S., Willie, C. K., \& Ainslie, P. N. Determinants of human cerebral pressure-flow velocity relationships: new insights from vascular modelling and $\mathrm{Ca}(2)(+)$ channel blockade. J. Physiol. 589, 3263-3274, doi:10.1113/jphysiol.2011.206953 jphysiol.2011.206953 [pii] (2011).

18. Zhang, R. et al. Autonomic neural control of dynamic cerebral autoregulation in humans. Circulation. 106, 1814-1820 (2002).

19. Tan, C. O. Defining the characteristic relationship between arterial pressure and cerebral flow. J. Appl. Physiol. 113, 1194-1200, doi:10.1152/ japplphysiol.00783.2012 japplphysiol.00783.2012 [pii] (2012).

20. Schmidt, E. A. et al. Symmetry of cerebral hemodynamic indices derived from bilateral transcranial Doppler. J. Neuroimaging. 13, 248-254 (2003).

21. Paulson, O. B., Strandgaard, S., \& Edvinsson, L. Cerebral autoregulation. Cerebrovasc. Brain. Metab. Rev. 2, 161-192 (1990).

22. Hamner, J. W., Morin, R. J., Rudolph, J. L., \& Taylor, J. A. Inconsistent link between low-frequency oscillations: R-R interval responses to augmented Mayer waves. J. Appl. Physiol. 90, 1559-1564 (2001).

23. Hamner, J. W., Tan, C. O., Tzeng, Y. C., \& Taylor, J. A. Cholinergic control of the cerebral vasculature in humans. J. Physiol. 590, 6343-6352, doi:10.1113/jphysiol.2012.245100 jphysiol.2012.245100 [pii] (2012).

24. Hamner, J. W., Tan, C. O., Lee, K., Cohen, M. A., \& Taylor, J. A. Sympathetic control of the cerebral vasculature in humans. Stroke. 41 102-109, doi:10.1161/STROKEAHA.109.557132 STROKEAHA.109.557132 [pii] (2010).

25. Narayanan, K., Collins, J. J., Hamner, J., Mukai, S., \& Lipsitz, L. A. Predicting cerebral blood flow response to orthostatic stress from resting dynamics: effects of healthy aging. Am. J. Physiol. Regul. Integr. Comp. Physiol. 281, R716-722 (2001).

26. Lieshout, J. J., \& Secher, N. H. Point:Counterpoint: Sympathetic activity does/does not influence cerebral blood flow. Point: Sympathetic activity does influence cerebral blood flow. J. Appl. Physiol. 105, 1364-1366, doi:10.1152/japplphysiol.90597.2008 90597.2008 [pii] (2008).

27. Taylor, J. A., Carr, D. L., Myers, C. W., \& Eckberg, D. L. Mechanisms underlying very-low-frequency RR-interval oscillations in humans. Circulation. 98, 547-555 (1998).

28. Giller, C. A., \& Mueller, M. Linearity and non-linearity in cerebral hemodynamics. Med. Eng. Phys. 25, 633-646, doi:S1350453303000286 [pii] (2003).

29. Friedman, J. H., \& Stuetzle, W. Projection pursuit regression. Am. Stat. Assoc. 76, 817-823 (1981).

30. Friedman, J. H., \& Tukey, J. W. A projection pursuit algorithm for exploratory data analysis. IEEE Trans. Comp. 23, 881-889 (1974).

31. Claassen, J. A., Levine, B. D., \& Zhang, R. Dynamic cerebral autoregulation during repeated squat-stand maneuvers. J. Appl. Physiol. (1985). 106, 153-160, doi:10.1152/japplphysiol.90822.2008 90822.2008 [pii] (2009).

32. Taylor, J. A., Myers, C. W., Halliwill, J. R., Seidel, H., \& Eckberg, D. L. Sympathetic restraint of respiratory sinus arrhythmia: implications for vagal-cardiac tone assessment in humans. Am. J. Physiol. Heart Circ. Physiol. 280, H2804-2814 (2001).

33. Aries, M. J., Elting, J. W., De Keyser, J., Kremer, B. P., \& Vroomen, P. C. Cerebral autoregulation in stroke: a review of transcranial Doppler studies. Stroke. 41, 2697-2704, doi:10.1161/STROKEAHA.110.594168STROKEAHA.110.594168 [pii] (2010).

34. Rangel-Castilla, L., Gasco, J., Nauta, H. J., Okonkwo, D. O., \& Robertson, C. S. Cerebral pressure autoregulation in traumatic brain injury. Neurosurg. Focus. 25, E7, doi:10.3171/FOC.2008.25.10.E7 (2008). 